\title{
Response: Commentary: Skilled Bimanual Training Drives Motor Cortex Plasticity in Children with Unilateral Cerebral Palsy
}

\author{
Ya-Ching Hung ${ }^{1 *}$, Kathleen M. Friel ${ }^{2}$ and Andrew M. Gordon ${ }^{3}$ \\ ${ }^{1}$ Family, Nutrition, and Exercise Sciences, Queens College, City University of New York, Flushing, NY, United States, \\ ${ }^{2}$ Neurology and Neuroscience, Burke Medical Research Institute, White Plains, NY, United States, ${ }^{3}$ Biobehavioral Sciences, \\ Teachers College, Columbia University, New York, NY, United States
}

Keywords: pediatrics, rehabilitation, transcranial magnetic stimulation, kinematics, neuroplasticity, hemiplegia

\section{A commentary on}

Commentary: Skilled Bimanual Training Drives Motor Cortex Plasticity in Children with Unilateral Cerebral Palsy

by Serrien, D. J. (2017). Front. Hum. Neurosci. 11:297. doi: 10.3389/fnhum.2017.00297

\section{OPEN ACCESS}

Edited by:

Mikhail Lebedev,

Duke University, United States

Reviewed by:

Douglas Owen Cheyne,

Hospital for Sick Children, Canada

*Correspondence:

Ya-Ching Hung

yaching.hung@qc.cuny.edu

Received: 15 November 2017 Accepted: 06 December 2017 Published: 15 December 2017

Citation:

Hung Y-C, Friel KM and Gordon AM

(2017) Response: Commentary:

Skilled Bimanual Training Drives Motor

Cortex Plasticity in Children with

Unilateral Cerebral Palsy.

Front. Hum. Neurosci. 11:619.

doi: 10.3389/fnhum.2017.00619
We thank Dr. Serrien for her interest in our paper and for the opportunity to clarify the differences between using clinical measures and kinematic measures to assess bimanual proficiency changes in children with unilateral spastic cerebral palsy (USCP). The primary clinical measure in Friel et al. (2016), the Assisting Hand Assessment (AHA), quantifies the quality of spontaneous use of the more-affective hand during bimanual activities. The AHA is validated and represents "expected outcomes" to quantify meaningful changes in functional ability at the International Classification of Functioning, Disability and Health (ICF) activity level, which describes human functioning including both capacity and performance. We concur that measures that assess bimanual coordination patterns in relation to individual task components could provide additional insights about bimanual coordination pattern strengthening following training. Dr. Serrien has been at the forefront of quantifying bimanual coordination (e.g., Serrien, 2008). She used tasks such as a bimanual drawer-opening task, in which one hand opens a pull-drawer and the other hand manipulates its contents, that she adapted with Dr. Mario Wiesendanger (Serrien and Wiesendanger, 2001) from his non-human primate studies (Kazennikov et al., 1994). We have adapted this task to quantify bimanual coordination in children with USCP and demonstrated specific deficits in bimanual spatio-temporal control compared to typically-developing children (Hung et al., 2004, 2010). Children with USCP were found to have reduced movement overlap of the two hands (i.e., moved each hand more sequentially) and thus longer durations between completion of the goal for each hand (drawer-opening and content manipulation, respectively). In fact, this assessment was sensitive in determining differences between unimanual and bimanual intensive treatments (Hung et al., 2011) while clinical outcomes were not (Gordon et al., 2011). Importantly, in a subset of participants in the Friel et al. (2016) study, kinematics during the same bimanual drawer-opening task were in fact subsequently examined (Hung et al., 2017). While there were no group AHA differences between the structured skill and unstructured practice groups, kinematic differences in bimanual drawer-opening were observed. Specifically, children in the structured skill group showed improvements in trunk and elbow movement control after training. Such improvements in more affected upper extremity movement control may correspond with the increase in motor map size of the more affected hand (Friel et al., 2016). Interestingly, no group differences were found for temporal measurements in the bimanual coordination pattern (both 
groups improved), while there were differences between the two groups in motor map changes (i.e., the structured skill group had expansion of the M1 motor map whereas the unstructured practice group did not).

Dr. Serrien correctly noted that the changes in cortical plasticity for the structured practice group were independent of the hemisphere of control, and the fact that both hemispheres respond to the bimanual training is in contrast with the findings of Kuhnke et al. (2008), who showed that functional gains are less pronounced in children with ipsilateral than contralateral control of the affected hand following unimanual training (constraintinduced movement therapy). While we agree that it is essential that rehabilitation approaches are optimized across the pathology of USCP, we should point out that we have shown that children with ipsilateral and children with contralateral control of the affected hand show similar levels of improvement following bimanual training (Smorenburg et al., 2017). One could argue that the current TMS analysis examined only the more affected hand, rather than areas associated with bimanual movement (Serrien, 2017). Friel et al. (2016) suggested that plasticity may have occurred in other brain regions. Recently we showed that structure (using diffusion tensor imaging) and function (using electroencephalography) of the somatosensory system is also highly related to the magnitude of a child's hand movement impairment (Gupta et al., 2017). Although we do not yet know how the somatosensory system may change after intensive hand therapy, the tight association between sensory system function and hand impairment suggests that sensory system plasticity may also underlie the improvements produced by intensive

\section{REFERENCES}

Friel, K. M., Kuo, H. C., Fuller, J., Ferre, C. L., Brandão, M., Carmel, J. B., et al. (2016). Skilled bimanual training drives motor cortex plasticity in children with unilateral cerebral palsy. Neurorehabil. Neural Repair 30, 834-844. doi: $10.1177 / 1545968315625838$

Gordon, A. M., Hung, Y. C., Brandao, M., Ferre, C. L., Kuo, H. C., Friel, K., et al. (2011). Bimanual training and constraint-induced movement therapy in children with hemiplegic cerebral palsy: a randomized trial. Neurorehabil. Neural Repair. 25, 692-702. doi: 10.1177/1545968311402508

Gupta, D., Barachant, A., Gordon, A. M., Ferre, C., Kuo, H. C., Carmel, J. B., et al. (2017). Effect of sensory and motor connectivity on hand function in pediatric hemiplegia. Ann. Neurol. 82, 766-780. doi: 10.1002/ana.25080

Hung, Y. C., Brandão, M. B., and Gordon, A. M. (2017). Structured skill practice during intensive bimanual training leads to better trunk and arm control than unstructured practice in children with unilateral spastic cerebral palsy. Res. Dev. Disabil. 60, 65-76. doi: 10.1016/j.ridd.2016.11.012

Hung, Y. C., Casertano, L., Hillman, A., and Gordon, A. M. (2011). The effect of training specificity on bimanual coordination in children with hemiplegia. Res. Dev. Disabil. 32, 2724-2731. doi: 10.1016/j.ridd.2011.05.038

Hung, Y. C., Charles, J., and Gordon, A. M. (2004). Bimanual coordination during a goal-directed task in children with hemiplegic cerebral palsy. Dev. Med. Child. Neurol. 46, 746-753. doi: 10.1111/j.1469-8749.2004.tb00994.x

Hung, Y. C., Charles, J., and Gordon, A. M. (2010). The influence of task constraints in bimanual coordination for children with hemiplegic cerebral palsy. Exp. Bain Res. 201, 421 - 428. doi: 10.1007/s00221-009-2049-1

Kazennikov, O., Wicki, U., Corboz, M., Hyland, B., Palmeri, A., Rouiller, E. M., et al. (1994). Temporal structure of a bimanual goal-directed movement sequence in monkeys. Eur. J. Neurosci. 6, 203-210. doi: 10.1111/j.1460-9568.1994.tb00262.x therapy. Assessing plasticity in other brain regions, however, often requires the use of techniques that are expensive and difficult to use with children, such as functional magnetic resonance imaging (fMRI). The compliance required of study participants for the collection of robust fMRI data is often beyond the capability of a child.

There are limitations associated with kinematic analyses. First, although they may be more sensitive to changes in movement coordination and strategy than clinical measures, unlike for gait, few upper extremity tasks are standardized with normative data. Furthermore, the cost and personnel required for this type of data collection/analysis limits widespread use in clinical research. Finally, although such analyses provide insights into spatio-temporal coordination, it puts the goal of normalizing movement at the forefront. In contrast, motor learning approaches such as bimanual training are aimed at increasing function, even when that requires compensation rather than normalization of movement. Nonetheless, we whole heartedly agree with Dr. Serrien that detailing the properties of motor circuits, including motor and sensory systems, along with interhemispheric connectivity, will further enhance the evaluation of rehabilitation interventions for USCP, specifically related to bimanual coordination. Our current work is aimed precisely at these goals.

\section{AUTHOR CONTRIBUTIONS}

Y-CH wrote the first draft of this commentary. KF and AG edited and contributed to the writing of the final draft.

Kuhnke, N., Juenger, H., Walther, M., Berweck, S., Mall, V., and Staudt, M. (2008). Do patients with congenital hemiparesis and ipsilateral corticospinal projections respond differently to constraint-induced movement therapy? Dev. Med. Child Neurol. 50, 898-903. doi: 10.1111/j.1469-8749.2008. 03119.x

Serrien, D. J. (2008). Coordination constraints during bimanual vs. unimanual performance conditions. Neuropsychologia 46, 419-425. doi: 10.1016/j.neuropsychologia.2007.08.011

Serrien, D. J. (2017). Commentary: skilled bimanual training drives motor cortex plasticity in children with unilateral cerebral palsy. Front. Hum. Neurosci. 11:297. doi: 10.3389/fnhum.2017.00297

Serrien, D. J., and Wiesendanger, M. (2001). Dissociation of grip/load-force coupling during a bimanual manipulative assignment. Exp. Brain Res. 136, 417-420. doi: 10.1007/s002210000620

Smorenburg, A. R., Gordon, A. M., Kuo, H. C., Ferre, C. L., Brandao, M., Bleyenheuft, Y., et al. (2017). Does corticospinal tract connectivity influence the response to intensive bimanual therapy in children with unilateral cerebral palsy? Neurorehabil. Neural Repair 31, 250-260. doi: $10.1177 / 1545968316675427$

Conflict of Interest Statement: The authors declare that the research was conducted in the absence of any commercial or financial relationships that could be construed as a potential conflict of interest.

Copyright (c) 2017 Hung, Friel and Gordon. This is an open-access article distributed under the terms of the Creative Commons Attribution License (CC BY). The use, distribution or reproduction in other forums is permitted, provided the original author(s) or licensor are credited and that the original publication in this journal is cited, in accordance with accepted academic practice. No use, distribution or reproduction is permitted which does not comply with these terms. 